\title{
Caries de la primera infancia asociada a las inadecuadas prácticas de lactancia materna y uso de biberón. Revisión de la literatura
}

\section{Breastfeeding and bottle feeding and their relationship with caries in early childhood. Literature review}

\author{
Alex Carrera Robalino ${ }^{1}$, Katherine León Hildalgo ${ }^{1}$, Maria Ullaguari Landeta ${ }^{3}$, Alexander Cruz Gallegos ${ }^{4}$, Ana Armas Vega ${ }^{5}$
}

\section{Resumen}

Introducción: La caries de la infancia temprana (CIT) constituye un problema de salud de interés mundial. EI propósito de realizar una revisión de la literatura científica es mostrar cómo las prácticas inadecuadas de alimentación por lactancia materna y uso del biberón contribuyen a la caries de la primera infancia. Materiales y métodos: Se realizó una revisión sistemática, en línea, de la literatura existente en el período comprendido entre 2012 y 2018 en la base de datos Medline, empleando como palabras clave: Caries, Breastfeeding, Bottle feeding y Childhood. Resultados: Se obtuvieron 61 artículos, de los cuales 25 fueron contemplados en esta revisión por considerase afines al objetivo de la misma. La literatura revisada evidenció relación directa entre la presencia de CIT y la forma de alimentación, sin diferencia entre los tipos considerados. Conclusiones: El amamantamiento prolongado después de los dos años de edad del infante al igual que el uso de biberón adicionado con azúcares y carbohidratos constituye un riesgo directo para la CIT.

Palabras clave Caries, Lactancia, Niño, Alimentación Artificial, Conducta en la Lactancia. (Fuente: DeCS BIREME)

\section{ABSTRACT}

Introduction: Early childhood caries (ECC) is a health problem of worldwide interest. The purpose of this literature review is showed how an inadequate feeding practices by breastfeeding and bottle feeding contribute to early childhood caries. Materials and methods: A systematic online review of the existing literature from 2012 to 2018 was performed in the Medline database, using key words such as Caries, Breastfeeding, Bottle feeding and Childhood. Results: 61 articles were obtained, of which, 25 were considered in this review because they are considered to be related to the objective of this review. The literature reviewed showed a direct relationship between the presence of CIT and the way of feeding, with no difference between the types considered. Conclusions: Prolonged breastfeeding more than two years of age of the infant as well as the use of bottles added with sugars and carbohydrates constitute a direct risk for the CIT.

Key words Caries, Lactation, Child, Bottle feeding, Sucking Behavior. (Source: MeSH NLM)

${ }^{1}$ Universidad Tecnológica Equinoccial

Correspondencia:

Od. Alex Carrera alexcr199415@gmail.com
Este es un artículo Open Access distribuido bajo la licencia Creative Commons Atribución-NoComercialCompartir Igual 4.0

Citar como: Carrera A, León K, Ullaguari M, Cruz A. Armas A. Caries de la primera infancia asociada a las inadecuadas prácticas de lactancia materna y uso de biberón. Revisión de la literatura. KIRU. 2018; oct-dic; 15(4): 208-215 https://doi.org/10.24265/kiru.2018.v15n4.08 


\section{INTRODUCCIÓN}

La caries dental, considerada un problema de salud pública que afecta a toda la población independiente de la edad (1), muestra un avance rápido ${ }^{(2)}$ en la dentición decidua debido a las características histológicas de esta última ${ }^{(3)}$, desencadenando extensa pérdida de tejido ${ }^{(1)}$, que se inicia con cambios microbiológicos dentro del complejo biofilm ${ }^{(3)}$, donde la influencia del flujo y composición salivar, la exposición al flúor y el consumo de azúcares de la dieta entre otros ${ }^{(4)(5)}$ dificultan su control.

La caries temprana o de la infancia temprana (CIT) constituye el término que identifica a la lesión cuando esta afecta a niños menores de 71 meses (1)(6)(7), afectando la calidad de vida de quien la padece por el intenso dolor, lo que desencadena una alimentación deficiente, sumado a las implicaciones psicológicas ${ }^{(4)}$, que a llevan a la CIT a ser incluida dentro de la clasificación internacional de enfermedades de la OMS $\left(\right.$ ICD-11) ${ }^{(7)}$.

Su aparición guarda relación con diversos factores directos que incluyen la presencia de placa, la mala higiene bucal, el aumento de la edad, la frecuencia, duración y el momento de consumo de bebidas azucaradas $^{(2)}$, a lo que se suman factores socioeconómicos y culturales $^{(8)}$, observando mayor prevalencia en países de ingresos económicos bajos ${ }^{(7)}$.

La lactancia materna es la manera natural de proveer a los infantes de nutrientes, necesarios para su crecimiento y

desarrollo ${ }^{(7)}$ y se constituye en una práctica beneficiosa para la salud sistémica del bebé, reduciendo su morbilidad, la disminución de infecciones gastrointestinales e inflamatorias de la piel. Estos beneficios llevan a considerar a la lactancia materna como la primera línea de defensa del recién nacido por la Organización Mundial de la Salud (OMS), que recomienda el amamantamiento exclusivo durante los primeros seis meses de vida, complementada con la ingesta de alimentos hasta los dos años de edad (1)(6)(7). Pese a esto, por motivos culturales, de salud de la madre e incluso del recién nacido ${ }^{(1)(5)}$, la alimentación a base de biberón constituye una opción frecuentemente adoptada.

Existe la tendencia de asociar la alimentación mediante biberón con la prevalencia de $\mathrm{CIT}^{(1)(4)}$ y el alto contenido en azúcares(3)(7) asociados a esta; sin embargo, se encuentra bien documentado el hecho de que la educación preventiva basada en el conocimiento de las normas y prácticas resulta eficaz para evitar una intervención restauradora durante la primera infancia e incluso durante toda la vida ${ }^{(8)(9)(10)}$.

En el Ecuador, pese a los diferentes programas de salud preventiva implementados por los organismos de control, la caries dental sigue siendo un problema que afecta de manera significativa a lactantes y niños en edad preescolar (11)(12). Así, el objetivo de este estudio fue realizar una revisión de los reportes de investigación sobre la asociación entre las prácticas inadecuadas de alimentación de la lactancia materna prolongada y el uso de biberón con edulcorantes y la caries dental en la infancia.

\section{MATERIALES Y MÉTODOS}

Se realizó una revisión sistemática de literatura en la base de datos electrónica Medline, tomando en cuenta todos los artículos publicados en el periodo comprendido entre enero 2012 y abril de 2018, empleando como palabras clave: Caries, Breastfeeding, Bottle feeding y Childhood. Se planteó la pregunta de investigación a partir de la estrategia (PICO): Pacientes: niños de 0 a 6 años de edad; Intervención: exposición al factor de riesgo: la alimentación con biberón; Comparación: lactancia materna; Resultado: caries dental.

Los criterios de inclusión para el levantamiento de literatura fueron estudios de casos, ensayos clínicos, metanálisis y revisiones de literatura que presenten una evaluación en niños con dentición primaria hasta los 6 años que traten o comparen la lactancia materna y la alimentación con biberón en asociación con la CIT. Se obtuvo un total de 61 artículos, de los cuales se excluyeron aquellos que no cubrieron los elementos de búsqueda, quedando seleccionados solo 25 artículos.

\section{RESULTADOS}

La literatura revisada muestra al amamantamiento como un factor positivo, sin relación con $\mathrm{CIT}^{(1)(13)(14)}$, que no afecta el $\mathrm{pH}$ del biofilm bucal en lo que respecta a la progresión de caries ${ }^{(15)}$, catalogando como un factor protector a la progresión de caries durante los primeros 6-7 meses de vida del niño ${ }^{(5)(9)}$. Por otro lado, los autores revisados refieren una relación directa entre CIT y el amamantamiento prolongado (2)(3)(16)(17)(18)(19)(20), principalmente al ejecutado en horas de la noche, bajo libre demanda del niño ${ }^{(6,21,22,23)}$, y en asociación con su frecuencia ${ }^{(24)(25)}$, sobre todo cuando se prolonga por un lapso mayor a 6 meses $^{(26)}$ o en niños con una edad mayor a los 12 meses $^{(27)}$. 
Con respecto a la alimentación con biberón, tres artículos refieren un resultado positivo, al relacionarlo con CIT, sobre todo cuando higiene, alimentos azucarados ${ }^{(3)(1)(2)(8)(10)(28)(19)}$, consumo nocturno ${ }^{(22)(23)}$ y frecuencia ${ }^{(24)}$ se encuentran inmiscuidos. Un reducido número de autores descartan esta relación (14) (20). Tres artículos no asocian el

amamantamiento o el biberón como factores directos para la progresión de caries, destacando a los alimentos azucarados $^{(4)}$, duración de la alimentación, falta de una correcta higiene ${ }^{(29)}$, bajo peso al nacer del niño y el hábito de fumar de la madre ${ }^{(13)}$ como factores de mayor relevancia para la CIT.

La revisión de literatura realizada para cada uno de los artículos seleccionados se detalla en la Tabla 1.

Tabla 1. Resumen de estudios evaluados sobre leche materna y biberón y su relación con CIT.

\begin{tabular}{|c|c|c|c|c|}
\hline AUTOR & Tipo de Estudio & Muestra & OBJETIVO & CONCLUSIONES \\
\hline $\begin{array}{l}\text { Avila W. et } \\
\text { al., } 2015^{1}\end{array}$ & $\begin{array}{l}\text { Revisión } \\
\text { sistemática } \\
\text { Metanálisis. }\end{array}$ & $\begin{array}{l}\text { Estudios } \\
\text { observaciona-les } \\
\text { transversales, de } \\
\text { casos y controles y } \\
\text { de cohortes, junto } \\
\text { con ensayos } \\
\text { clínicos de niños } \\
\text { con dentición } \\
\text { exclusivamente } \\
\text { primaria (edad } \leq 71 \\
\text { meses). }\end{array}$ & $\begin{array}{l}\text { Buscar la evidencia } \\
\text { científica en } \\
\text { respuesta a la } \\
\text { alimentación con } \\
\text { biberón produce } \\
\text { más CIT que el } \\
\text { amamantamiento. }\end{array}$ & $\begin{array}{l}\text { La evidencia científica disponible mostró } \\
\text { que la lactancia materna es más eficaz en la } \\
\text { prevención de la caries dental en la infancia } \\
\text { temprana que la alimentación con biberón } \\
\text { (1). }\end{array}$ \\
\hline $\begin{array}{l}\text { Prakasha } \\
\text { Shrutha S. } \\
\text { et al., } 2013^{2}\end{array}$ & Ensayo Clínico. & $\begin{array}{l}\text { (974 niños y } 1026 \\
\text { niñas) niños de } 3-5 \\
\text { años de una } \\
\text { muestra aleatoria } \\
\text { de preescolares en } \\
\text { el distrito de } \\
\text { Kanpur, India. }\end{array}$ & $\begin{array}{l}\text { Evaluar la caries en } \\
\text { niños de } 3 \text { a } 5 \text { años } \\
\text { y la relación con las } \\
\text { diferentes formas de } \\
\text { alimentación de las } \\
\text { madres y los hábitos } \\
\text { higiénicos orales. }\end{array}$ & $\begin{array}{l}\text { La prevalencia de caries fue alta y } \\
\text { estadísticamente significativa ( }(P<0,05) \\
\text { entre los niños alimentados con leche } \\
\text { materna durante un período prolongado } \\
\text { durante la noche que aquellos que concilian } \\
\text { el sueño con un biberón (2). }\end{array}$ \\
\hline $\begin{array}{l}\text { Kato T. et } \\
\text { al., } 2015^{3}\end{array}$ & Ensayo Clínico. & $\begin{array}{l}43.383 \text { bebés a la } \\
\text { edad de } 6 \text { meses. }\end{array}$ & $\begin{array}{l}\text { Investigar la } \\
\text { asociación entre la } \\
\text { duración del } \\
\text { amamantamiento } \\
\text { en el primer } \\
\text { semestre de vida y } \\
\text { su riesgo de ClT } \\
\text { entre los } 30-66 \\
\text { meses de edad en } \\
\text { Japón. }\end{array}$ & $\begin{array}{l}\text { Se encontró que los niños que habían sido } \\
\text { alimentados con leche materna durante al } \\
\text { menos } 6 \text { o } 7 \text { meses, tanto en forma } \\
\text { exclusiva y parcial, estaban en riesgo } \\
\text { elevado de caries dental a la edad de } 30 \\
\text { meses, en comparación con los que habían } \\
\text { sido alimentados exclusivamente con } \\
\text { fórmula }{ }^{(3)} \text {. }\end{array}$ \\
\hline $\begin{array}{l}\text { Folayan M. } \\
\text { et al., } 2015^{4}\end{array}$ & Ensayo Clínico. & $\begin{array}{l}497 \text { niños de } 6 \\
\text { meses a } 71 \text { meses } \\
\text { quer fueron } \\
\text { reclutados a través } \\
\text { de una encuesta } \\
\text { de hogares } \\
\text { realizada en lle-Ife, } \\
\text { Nigeria. }\end{array}$ & $\begin{array}{lr}\text { Determinar } & \text { la } \\
\text { prevalencia } & \text { e } \\
\text { indicadores } & \text { de } \\
\text { riesgo de CIT en la } \\
\text { población } \\
\text { suburbana } \\
\text { Nigeria. }\end{array}$ & $\begin{array}{l}\text { Los programas que mejoren el conocimiento } \\
\text { de la salud oral de madres deben ser } \\
\text { capaces de dar lugar a la mejora de las } \\
\text { prácticas de higiene oral y la reducción del } \\
\text { consumo de alimentos azucarados entre las } \\
\text { comidas de los niños en edad preescolar (4). }\end{array}$ \\
\hline
\end{tabular}




\begin{tabular}{|c|c|c|c|c|}
\hline $\begin{array}{l}\text { Cidro J. et } \\
\text { al., } 2015^{8}\end{array}$ & Ensayo Clínico. & $\begin{array}{l}\text { Participantes del } \\
\text { estudio } \\
\text { Teeth Talk". }\end{array}$ & $\begin{array}{lr}\begin{array}{l}\text { Resaltar } \\
\text { importancia }\end{array} & \text { la } \\
\text { conocimiento } \\
\text { ancestral } \\
\text { transferido a la } \\
\text { higiene oral y las } \\
\text { practicas } \\
\text { alimenticias en los } \\
\text { infantes de las } \\
\text { comunidades } \\
\text { aborígenes. }\end{array}$ & $\begin{array}{l}\text { Tradiciones de alimentación infantil como la } \\
\text { lactancia materna son conocidas por ser } \\
\text { eficaces en la reducción de enfermedades } \\
\text { infantiles como la CIT. } \\
\text { La lactancia materna y la salud de los } \\
\text { dientes se ven unidas por los encuestados. } \\
\text { Los participantes eran conscientes de que } \\
\text { incluso cuando era leche o fórmula en la } \\
\text { botella, las técnicas de alimentación } \\
\text { inadecuadas y la falta de cuidado oral dieron } \\
\text { lugar a la caries dental (8). }\end{array}$ \\
\hline $\begin{array}{l}\text { Olatosi O. et } \\
\text { al., } 2015^{9}\end{array}$ & Ensayo Clínico. & $\begin{array}{l}\text { Se seleccionaron } \\
302 \text { niños de } 6-71 \\
\text { meses de cuatro } \\
\text { clínicas pediátricas } \\
\text { ambulatorias en } \\
\text { Lagos, Nigeria. }\end{array}$ & $\begin{array}{l}\text { Determinar la } \\
\text { prevalencia de CIT y } \\
\text { su asociación con la } \\
\text { alimentación infantil } \\
\text { y el conocimiento de } \\
\text { los hábitos orales en } \\
\text { preescolares de 6- } \\
71 \text { meses en Lagos. }\end{array}$ & $\begin{array}{l}\text { CIT fue significativamente mayor en los } \\
\text { niños que fueron alimentados con biberón } \\
\text { por la noche. La lactancia materna mostró } \\
\text { significativamente menor prevalencia de } \\
\text { caries }{ }^{(9)} \text {. }\end{array}$ \\
\hline $\begin{array}{l}\text { Nakayama } \\
\text { Y. et al., } \\
2015^{21}\end{array}$ & Ensayo Clínico & $\begin{array}{llr}\text { Los sujetos } & \text { de } \\
\text { estudio } & \text { fueron } \\
1675 \text { niños } & \text { de } \\
\text { entre } 18 \text { y } & 23 \\
\text { meses. } & & \end{array}$ & $\begin{array}{l}\text { Investigar la } \\
\text { asociación entre } \\
\text { amamantamiento } \\
\text { nocturno, bocadillos } \\
\text { nocturnos y otros } \\
\text { factores de riesgo y } \\
\text { CIT en niños } \\
\text { japoneses de 18-23 } \\
\text { meses de edad. }\end{array}$ & $\begin{array}{l}\text { Este estudio sugiere que los hábitos de } \\
\text { lactancia materna y bocadillos nocturnos } \\
\text { están correlacionados con la CIT }{ }^{(21)} \text {. }\end{array}$ \\
\hline $\begin{array}{l}\text { Chaffee B. } \\
\text { et } \quad \text { al., } \\
2014^{26}\end{array}$ & Ensayo Clínico & $\begin{array}{l}715 \text { familias de } \\
\text { bajos ingresos en } \\
\text { Portor Alegre, } \\
\text { Brasil. Se comparó } \\
\text { la prevalencia de } \\
\text { CIT en edad de } 38 \\
\text { meses. }\end{array}$ & $\begin{array}{l}\text { Estimar la } \\
\text { asociación entre } \\
\text { amamantamiento } \\
\text { mayor a } 24 \text { meses y } \\
\text { CIT severa. }\end{array}$ & $\begin{array}{l}\text { En esta población, la lactancia materna en } \\
\text { niños } \geq 24 \text { meses, sobre todo si es } \\
\text { frecuente, se asoció con graves casos de } \\
\text { CIT en comparación con la lactancia } \\
\text { materna }<6 \text { meses }{ }^{(26)} \text {. }\end{array}$ \\
\hline $\begin{array}{l}\text { Corrêa- } \\
\text { Faria P. et } \\
\text { al., } 2013^{29}\end{array}$ & Ensayo Clínico & $\begin{array}{l}593 \text { niños de entre } \\
\text { tres y cinco años. }\end{array}$ & $\begin{array}{lr}\text { Evaluar } & \text { la } \\
\text { asociación entre } \\
\text { polimorfismo en } \\
\text { genes MTR y } \\
\text { con riesgo de CIT y } \\
\text { alteraciones en el } \\
\text { índice de masa } \\
\text { corporal. }\end{array}$ & $\begin{array}{l}\text { CIT se asoció significativamente con la } \\
\text { edad, la duración de la lactancia materna, y } \\
\text { la higiene bucal }{ }^{(29)} \text {. }\end{array}$ \\
\hline $\begin{array}{l}\text { Prakash P. } \\
\text { et } \quad \text { al., } \\
2012^{22}\end{array}$ & Ensayo Clínico & $\begin{array}{l}\text { Una muestra } \\
\text { aleatoria de } 1.500 \\
\text { niños de edades } \\
\text { comprendidas } \\
\text { entre } 8 \text { y } 48 \text { meses } \\
\text { se seleccionó de } \\
\text { diversas partes de } \\
\text { la ciudad de } \\
\text { Bangalore. }\end{array}$ & $\begin{array}{lr}\text { Investigar } & \text { la } \\
\text { prevalencia y los } \\
\text { factores de } & \text { riesgo } \\
\text { relacionados a } & \text { CIT } \\
\text { en } & \text { niños } \\
\text { preescolares } & \text { en } \\
\text { India. } & \end{array}$ & $\begin{array}{l}\text { Un aumento significativo en la prevalencia } \\
\text { de caries fue encontrado en niños } \\
\text { acostumbrados a la práctica de la lactancia } \\
\text { materna a demanda y la alimentación con } \\
\text { biberón por la noche }{ }^{(22)} \text {. }\end{array}$ \\
\hline $\begin{array}{l}\text { Stephen A. } \\
\text { et } \quad \text { al., } \\
2017^{23}\end{array}$ & Ensayo Clínico & $\begin{array}{l}\text { Niños de entre tres } \\
\text { y seis años de } \\
\text { edad, } \\
\text { seleccionados al } \\
\text { azar de escuelas } \\
\text { en escuelas } \\
\text { semiurbanas, } \\
\text { urbanas y rurales } \\
\text { en Salem. }\end{array}$ & $\begin{array}{l}\text { Evaluar la } \\
\text { asociación entre CIT } \\
\text { y los factores de } \\
\text { riesgo entre los } 3 \text { a } 6 \\
\text { años, de las } \\
\text { escuelas rurales, } \\
\text { semi rurales y } \\
\text { urbanas en India. }\end{array}$ & $\begin{array}{l}\text { Existió una relación significativa entre } \\
\text { amamantamiento y demanda libre, duración } \\
\text { y frecuencia por más de } 12,18 \text { y } 24 \text { meses. } \\
\text { Existió una relación significativa entre CIT y } \\
\text { uso de biberón, alimentos azucarados en } \\
\text { biberón y biberón nocturno }(p<0,05)(23) \text {. }\end{array}$ \\
\hline
\end{tabular}




\begin{tabular}{|c|c|c|c|c|}
\hline $\begin{array}{l}\text { Cui L. et al., } \\
2017^{27}\end{array}$ & Metanálisis & $\begin{array}{l}35 \text { estudios que } \\
\text { involucran } 73,041 \\
\text { participantes de } 0- \\
71 \text { meses fueron } \\
\text { incluidos. }\end{array}$ & $\begin{array}{l}\text { Evaluar } r \text { la } \\
\text { asociación entre } \\
\text { amamantamiento y } \\
\text { riesgo de CIT. }\end{array}$ & $\begin{array}{l}\text { El amamantamiento exclusivo no tiene una } \\
\text { disminución significativa ( } p=0,248) \text { del } \\
\text { riesgo de CIT en comparación con el uso de } \\
\text { biberón. } \\
\text { Amamantamiento mayor a } 12 \text { meses } \\
\text { incrementa el riesgo de CIT en comparación } \\
\text { a un amamantamiento menor a } 12 \text { meses } \\
\text { ( } p<0,001) \text {. } \\
\text { Se encontró una asociación } \\
\text { significativamente inversa entre solo una } \\
\text { alimentación por amamantamiento y el } \\
\text { riesgo de CIT y menor a } 12 \text { meses entre los } \\
3 \text { a } 6 \text { años (27). }\end{array}$ \\
\hline $\begin{array}{l}\text { Kraljevic I. } \\
\text { et } \\
2017^{16} \text { al., }\end{array}$ & Ensayo Clínico & $\begin{array}{l}82 \text { padres de niños } \\
\text { escogidos entre } \\
2010 \text { y } 2014\end{array}$ & $\begin{array}{lr}\text { Determinar } & \text { los } \\
\text { indicadores de } \\
\text { riesgo y su } \\
\text { correlación en niños } \\
\text { con alta prevalencia } \\
\text { de caries y } \\
\text { necesidad alta de } \\
\text { tratamiento. }\end{array}$ & $\begin{array}{l}\text { El amamantamiento y el uso de biberón } \\
\text { actúan como cofactor de riesgo para } \\
\text { producir CIT (16). }\end{array}$ \\
\hline $\begin{array}{ll}\text { Kakanur } & \text { M. } \\
\text { et } & \text { al., } \\
2017^{17} & \end{array}$ & Ensayo Clínico & $\begin{array}{l}\text { Muestra aleatoria } \\
\text { de } 1152 \text { niños }\end{array}$ & $\begin{array}{lr}\text { Prevalencia } & y \\
\text { asociación de los } \\
\text { factores de riesgo } \\
\text { de CIT en niños } \\
\text { preescolares entre } \\
\begin{array}{l}\text { 3-5 años en la } \\
\text { cuidad } \\
\text { Bengaluru. }\end{array} \\
\end{array}$ & $\begin{array}{l}\text { Se encontró una relación estadísticamente } \\
\text { significativa entre el riesgo de CIT y } \\
\text { amamantamiento. Aunque su evidencia no } \\
\text { sustenta un fuerte y consistente relación } \\
\text { entre amamantamiento y riesgo de CIT (17). }\end{array}$ \\
\hline $\begin{array}{l}\text { Majorana A. } \\
\text { et } \quad \text { al., } \\
2014^{18}\end{array}$ & Ensayo Clínico & $\begin{array}{lr}2395 & \text { niños } \\
\text { italianos de } & 24-30 \\
\text { meses } & \end{array}$ & $\begin{array}{l}\text { Investigar la } \\
\text { asociación entre las } \\
\text { prácticas } \\
\text { alimenticias, } \\
\text { amamantamiento, } \\
\text { exposición y SES } \\
\text { ambiente y SES de } \\
\text { como factores el } \\
\text { riesgo en elles } \\
\text { desarrollo de caries } \\
\text { en niños de 24-30 } \\
\text { meses de edad. }\end{array}$ & $\begin{array}{l}\text { El amamantamiento, frecuencia, duración, y } \\
\text { realizarlo en la noche tiene una asociación } \\
\text { positiva con CIT, aunque concluyen que la } \\
\text { CIT se produce por varios factores } \\
\text { adicionales (18). }\end{array}$ \\
\hline $\begin{array}{l}\text { Olatosi O. et } \\
\text { al., } 2014^{10}\end{array}$ & Ensayo Clínico & $\begin{array}{l}302 \text { niños fueron } \\
\text { incluidos en este } \\
\text { estudio. }\end{array}$ & $\begin{array}{l}\text { Determinar la } \\
\text { asociación de CIT } \\
\text { con amamanta- } \\
\text { miento } \\
\text { alimentación y con } \\
\text { biberón en niños de } \\
\text { edad preescolar. }\end{array}$ & $\begin{array}{l}\text { El amamantamiento en los primeros } 3 \text { a } 6 \\
\text { meses de vida está asociado con un bajo } \\
\text { índice de caries dental mientras que el uso } \\
\text { de biberón en el día y en la noche está } \\
\text { asociado con un alto índice de CIT (10). }\end{array}$ \\
\hline $\begin{array}{l}\text { Çolak H. et } \\
\text { al., } 2013^{28}\end{array}$ & $\begin{array}{l}\text { Artículo } \\
\text { revisión }\end{array}$ & $\begin{array}{lr}149 \quad \text { artículos } \\
\text { revisados } & \text { y } \\
\text { referenciados. }\end{array}$ & $\begin{array}{l}\text { Actualización sobre } \\
\text { causas, diagnóstico } \\
\text { y tratamiento sobre } \\
\text { CIT. }\end{array}$ & $\begin{array}{l}\text { Existe una relación entre CIT y biberón y } \\
\text { dormir con un biberón, el amamantamiento } \\
\text { ideal provee una perfecta nutrición al } \\
\text { infante, ya que un amamantamiento } \\
\text { nocturno y prolongado aumenta el riesgo de } \\
\text { CIT, especialmente después de } 12 \text { meses } \\
\text { (28). }\end{array}$ \\
\hline
\end{tabular}




\begin{tabular}{|c|c|c|c|c|}
\hline $\begin{array}{l}\text { Bernabé } \text { E. } \\
\text { et al., } \\
2017^{13}\end{array}$ & Estudio Clínico & $\begin{array}{l}1102 \text { niños fueron } \\
\text { incluidos en este } \\
\text { análisis }\end{array}$ & $\begin{array}{l}\text { Evidenciar los } \\
\text { efectos de recién } \\
\text { nacidos con bajo } \\
\text { peso,amamantamie } \\
\text { nto y uso de tabaco } \\
\text { en el embarazo para } \\
\text { la formación de CIT. }\end{array}$ & $\begin{array}{l}\text { Niños con bajo peso al nacer y madres } \\
\text { fumadoras presentaron mayor presencia de } \\
\text { caries que niños con peso normal y madre } \\
\text { no fumadoras. No existió diferencias entre } \\
\text { los dos grupos sobre la duración del } \\
\text { amamantamiento (13). }\end{array}$ \\
\hline $\begin{array}{l}\text { Neves P. et } \\
\text { al., } 2016^{15}\end{array}$ & Estudio Clínico & $\begin{array}{l}\text { Se expusieron } \\
\text { biofilm de } 16 \text { niños } \\
\text { ( } 7 \text { con CIT, } 9 \text { sin } \\
\text { caries). }\end{array}$ & $\begin{array}{l}\text { Evaluar la acidez de } \\
\text { la leche humana en } \\
\text { los biofilm de los } \\
\text { niños con y sin CIT. }\end{array}$ & $\begin{array}{l}\text { El amamantamiento no provocó una } \\
\text { disminución significativa de los niveles de } \\
\mathrm{pH} \text { en el biofilm del niño con o sin presencia } \\
\text { de CIT, sugiriendo que otros productos de la } \\
\text { dieta son los causantes de caries }{ }^{(15)} \text {. }\end{array}$ \\
\hline $\begin{array}{l}\text { Özen B. et } \\
\text { al., } 2016^{14}\end{array}$ & Estudio Clínico & $\begin{array}{l}408 \text { niños en edad } \\
\text { preescolar que } \\
\text { fueron } \\
\text { seleccionados al } \\
\text { azar de un total de } \\
4116 \text { niños. } \\
\text { Grupo de niños de } \\
24 \text { a } 71 \text { meses. }\end{array}$ & $\begin{array}{l}\text { Evaluar los factores } \\
\text { asociados para el } \\
\text { desarrollo de CIT y } \\
\text { S- CIT. }\end{array}$ & $\begin{array}{l}\text { El uso de biberón o amamantamiento no } \\
\text { tuvo una relación estadísticamente } \\
\text { significativa con CIT (14). }\end{array}$ \\
\hline $\begin{array}{l}\text { Gopal S. et } \\
\text { al., } 2016^{19}\end{array}$ & Estudio clínico & $\begin{array}{l}477 \text { niños en edad } \\
\text { preescolar de } 3 \text { a } 6 \\
\text { años fueron } \\
\text { seleccionados al } \\
\text { azar de las } \\
\text { escuelas en el área } \\
\text { de estudio. }\end{array}$ & $\begin{array}{l}\text { Determinar la } \\
\text { prevalencia y el } \\
\text { potencial riesgo de } \\
\text { CIT en niños de } 3 \text { a } \\
6 \text { años. }\end{array}$ & $\begin{array}{l}\text { CIT es más prevalente en niños } \\
\text { acostumbrados a solicitar el a el } \\
\text { amamantamiento a cualquier hora, biberón } \\
\text { nocturno, consumo de golosinas y dulces } \\
\text { entre comidas }{ }^{(19)} \text {. }\end{array}$ \\
\hline $\begin{array}{ll}\text { Feldens } & \text { C. } \\
\text { et } & \text { al., } \\
2018^{24} & \end{array}$ & Estudio clínico & $\begin{array}{ll}345 & \text { niños } \\
\text { brasileños. }\end{array}$ & $\begin{array}{ll}\text { Investigar } & \text { la } \\
\text { asociación entre la } \\
\text { frecuencia de } \\
\text { amamantamiento } \\
\text { por } 12 \text { meses y la } \\
\text { prevalencia de } \\
\text { caries. }\end{array}$ & $\begin{array}{l}\text { La alta frecuencia de alimentación, ya sea } \\
\text { por biberón o amamantamiento, está } \\
\text { asociada positivamente con CIT (24). }\end{array}$ \\
\hline $\begin{array}{l}\text { Peres K. et } \\
\text { al., } 2017^{25}\end{array}$ & Ensayo clínico & $\begin{array}{l}1303 \text { niños del sur } \\
\text { de Brasil. }\end{array}$ & $\begin{array}{l}\text { Investigar si la } \\
\text { duración del } \\
\text { amamantamiento es } \\
\text { un riesgo para la } \\
\text { CIT. }\end{array}$ & $\begin{array}{l}\text { Amamantamiento prolongado aumenta el } \\
\text { riesgo de CIT }{ }^{(25)} \text {. }\end{array}$ \\
\hline $\begin{array}{l}\text { Sun X. et } \\
\text { al., } 2017^{20}\end{array}$ & Ensayo Clínico & $\begin{array}{l}\text { Se analizaron los } \\
\text { datos de } 9722 \\
\text { niños en edad } \\
\text { preescolar que } \\
\text { participaron en la } \\
\text { tercera Encuesta } \\
\text { Nacional de Salud } \\
\text { Oral de China. }\end{array}$ & $\begin{array}{l}\text { Explorar la } \\
\text { asociación entre los } \\
\text { factores de la vida } \\
\text { temprana y caries } \\
\text { en niños de } 5 \text { años }\end{array}$ & $\begin{array}{l}\text { Niños que solo utilizaron biberón } \\
\text { presentaron menor asociación que los niños } \\
\text { que recibieron amamantamiento (20). }\end{array}$ \\
\hline $\begin{array}{l}\text { Birungi N. et } \\
\text { al., } 2017^{5}\end{array}$ & Ensayo Clínico & $\begin{array}{l}417 \text { parejas de } \\
\text { madres e hijos. }\end{array}$ & $\begin{array}{l}\text { Estimar el efecto de } \\
\text { CIT en niños de } 5 \\
\text { años en Uganda, } \\
\text { enfocándose en el } \\
\text { amamantamiento } \\
\text { exclusivo y su } \\
\text { relación con ClT. }\end{array}$ & $\begin{array}{l}\text { Un amamantamiento exclusivo durante las } \\
\text { primeras } 24 \text { semanas de vida del niño es un } \\
\text { factor protector ante CIT }{ }^{(5)} \text {. }\end{array}$ \\
\hline
\end{tabular}




\section{DISCUSIÓN}

La CIT se entiende como la lesión que afecta al bebé hasta los 6 años de vida ${ }^{(1)(6)(7)}$. Su presencia de forma tradicional ha sido asociada a prácticas dietéticas inadecuadas, uso de la biberón con líquidos azucarados (3)(5)(4) contenidos en un envase o botella, administrados de manera prolongada y frecuente en especial durante la noche (22)(23), donde su asociación con factores socioeconómicos, de entorno afectivo, de conocimiento y motivación con respecto a sus padres resulta definitiva para su prevención ${ }^{(30)}$.

La Academia Americana de Pediatría identifica la leche humana como el nutriente ideal para el recién nacido ${ }^{(6)}$ ), con múltiples ventajas relacionadas con la salud del bebé y de la madre ${ }^{(31)(32)}$. Sin embargo, resulta

inminente contar con procesos de capacitación y control de hábitos mediante procesos de capacitación y motivación dirigidos a los padres y/o responsables (22)(30) sin dejar de lado a los profesionales de la salud, médicos de familia, pediatras y ginecólogos, convirtiéndolos en agentes multiplicadores y evaluadores de los factores de riesgo, con el propósito de unificar esfuerzos y constituir una primera línea de cuidado al recién nacido(29)(30), consensuados desde la academia como elemento generador de conocimiento y formador del personal de salud; rompiendo paradigmas e idiosincrasias y desencadenando unión entre el personal de salud médico y odontólogo, que revierta frutos sobre la salud del joven paciente.

La educación y el conocimiento direccionado a los padres $y / 0$ responsables se muestra como herramienta fundamental para la prevención y tratamiento de CIT (30) con la ejecución de técnicas de cepillado adecuadas y la consecuente eliminación de bacterias de boca de la madre (23)(27). Establecidas junto con guías nutricionales direccionadas desde el periodo gestacional ${ }^{(31)(33)}$, se convierten en herramienta clave para el éxito en el control de la enfermedad.

Una de las principales limitantes del estudio es la reducida literatura de estudios ejecutados en Latinoamérica. La existente falta de unificación metodológica dificulta obtener una visión integral. En ese contexto, si bien las entidades de salud en el Ecuador se encuentran en la búsqueda de mejorar las condiciones de la población infantil (34)(35), se requiere unificar esfuerzos para un seguimiento y evaluación de las estrategias hasta el momento desarrolladas a corto y largo plazo mediante estudios clínicos multicéntricos y multidisciplinarios.

Considerando la evidencia científica reportada en esta revisión, podemos sustentar que el amamantamiento prolongado a más de dos años de edad del infante al igual que el uso de biberón adicionado con azúcares y carbohidratos constituyen un riesgo directo para la CIT, sobre todo cuando estos no se encuentran regulados de la manera adecuada. Sumado a factores socioeconómicos, como son los hábitos alimenticios e higiénicos entre otros, que los padres de familia -y los cuidadores- les transmiten a los niños, llegan a ser vitales para la formación de CIT.

Contribuciones de autoría: ACR, KLH, MUL, ACG, AAV diseñaron el estudio, recopilaron, analizaron los datos. Redactaron y aprobaron el artículo todos los autores.

\section{Fuente de Financiamiento: Autofinanciado}

Conflicto de interés: Los autores declaramos no tener ningún interés de por medio.

\section{REFERENCIAS}

1. Avila WM, Pordeus IA, Paiva SM, Martins CC. Breast and Bottle Feeding as Risk Factors for Dental Caries: A Systematic Review and Meta-Analysis. PLoS One. 2015;10(11): e0142922.

2. Prakasha Shrutha S, Vinit GBG, Giri KY, Alam S. Feeding practices and early childhood caries: a cross-sectional study of preschool children in kanpur district, India. ISRN Dent. 2013; 2013: 275193.

3. Kato T, Yorifuji T, Yamakawa M, Inoue S, Saito K, Doi H, et al. Association of breast feeding with early childhood dental caries: Japanese population-based study. BMJ Open. 2015 Mar; 5(3): e006982.

4. Folayan MO, Kolawole KA, Oziegbe EO, Oyedele T, Oshomoji O V, Chukwumah NM, et al. Prevalence and early childhood caries risk indicators in preschool children in suburban Nigeria. BMC Oral Health. 2015 Jun;15:72.

5. Birungi N, Fadnes LT, Kasangaki A, Nankabirwa V, Okullo I, Lie SA, et al. Assessing causal effects of early life-course factors on early childhood caries in 5-year-old Ugandan children using directed acyclic graphs (DAGs): A prospective cohort study. Community Dent Oral Epidemiol. 2017 Dec; 45(6): 512-21.

6. Paglia L. Does breastfeeding increase risk of early childhood caries? European Journal of Paediatric Dentistry: Official Journal of European Academy of Paediatric Dentistry. Italy. 2015; Vol. 16: 173.

7. Phantumvanit $P$, Makino $Y$, Ogawa $H$, Rugg-Gunn A, Moynihan P, Petersen PE, et al. WHO Global Consultation on Public Health Intervention against Early Childhood Caries. Community Dent Oral Epidemiol. 2018; 46(3): 280-287. 
8. Cidro J, Zahayko L, Lawrence HP, Folster S, McGregor M, McKay K. Breast feeding practices as cultural interventions for early childhood caries in Cree communities. BMC Oral Health. 2015 Apr; 15: 49.

9. Olatosi OO, Inem V, Sofola OO, Prakash P, Sote EO. The prevalence of early childhood caries and its associated risk factors among preschool children referred to a tertiary care institution. Niger J Clin Pract. 2015; 18(4): 493-501.

10. Olatosi OO, Sote EO. Association of early childhood caries with breastfeeding and bottle feeding in southwestern nigerian children of preschool age. J West African Coll Surg. 2014; 4(1): 31-53.

11. Ruiz N. Estudio Epidemiológico de Salud Bucal en Escolares Fiscales menores de 15 años. Quito: Ministerio de Salud Pública; 1996.

12. OPS. Estudio epidemiológico nacional de salud bucal en menores de 15 años del Ecuador. Quito: MSP/OPS; 2010;

13. Bernabe E, MacRitchie H, Longbottom C, Pitts NB, Sabbah W. Birth Weight, Breastfeeding, Maternal Smoking and Caries Trajectories. J Dent Res. 2017 Feb; 96(2): 171-8.

14. Ozen B, Van Strijp AJP, Ozer L, Olmus H, Genc A, Cehreli SB. Evaluation of Possible Associated Factors for Early Childhood Caries and Severe Early Childhood Caries: A Multicenter Cross-Sectional Survey. J Clin Pediatr Dent. 2016; 40(2): 118-23.

15. Neves PAM, Ribeiro CCC, Tenuta LMA, Leitao TJ, Monteiro-Neto V, Nunes AMM, et al. Breastfeeding, Dental Biofilm Acidogenicity, and Early Childhood Caries. Caries Res. 2016; 50(3): 319-24.

16. Kraljevic I, Filippi C, Filippi A. Risk indicators of early childhood caries (ECC) in children with high treatment needs. Swiss Dent J. 2017; 127(5): 398-410.

17. Kakanur M, Nayak M, Patil SS, Thakur R, Paul ST, Tewathia N. Exploring the multitude of risk factors associated with early childhood caries. Indian J Dent Res. 2017; 28(1): 27-32.

18. Majorana A, Cagetti MG, Bardellini E, Amadori F, Conti G, Strohmenger $L$, et al. Feeding and smoking habits as acumulative risk factors for early childhood caries in toddlers, after adjustment for several behavioral determinants: a retrospective study. BMC Pediatr. 2014 Feb; 14: 45.

19. Gopal S, Chandrappa V, Kadidal U, Rayala C, Vegesna M. Prevalence and Predictors of Early Childhood Caries in 3to 6-year-old South Indian Children--A Cross-sectional Descriptive Study. Oral Health Prev Dent. 2016; 14(3): 267-73.

20. Sun X, Bernabe E, Liu X, Gallagher JE, Zheng S. Early life factors and dental caries in 5-year-old children in China. J Dent. 2017 Sep; 64: 73-9.

21. Nakayama $Y$, Mori M. Association between nocturnal breastfeeding and snacking habits and the risk of early childhood caries in 18- to 23-month-old Japanese children. J Epidemiol. 2015; 25(2): 142-7.

22. Prakash $\mathrm{P}$, Subramaniam $\mathrm{P}$, Durgesh $\mathrm{BH}$, Konde $\mathrm{S}$. Prevalence of early childhood caries and associated risk factors in preschool children of urban Bangalore, India: A cross-sectional study. Eur J Dent. 2012 Apr;6(2):141-52.

23. Stephen A, Krishnan R, Chalakkal P. The Association between Cariogenic Factors and the Occurrence of Early
Childhood Caries in Children from Salem District of India. J Clin Diagn Res. 2017 Jul; 11(7): ZC63-ZC66.

24. Feldens CA, Rodrigues PH, de Anastacio G, Vitolo MR, Chaffee BW. Feeding frequency in infancy and dental caries in childhood: a prospective cohort study. Int Dent J. 2018 Apr; 68(2): 113-21.

25. Peres KG, Nascimento GG, Peres MA, Mittinty MN, Demarco FF, Santos IS, et al. Impact of Prolonged Breastfeeding on Dental Caries: A Population-Based Birth Cohort Study. Pediatrics. 2017 Jul; 140(1).

26. Chaffee BW, Feldens CA, Vitolo MR. Association of longduration breastfeeding and dental caries estimated with marginal structural models. Ann Epidemiol. 2014 Jun; 24(6): 448-54.

27. Cui L, Li X, Tian Y, Bao J, Wang L, Xu D, et al. Breastfeeding and early childhood caries: a meta-analysis of observational studies. Asia Pac J Clin Nutr. 2017; 26(5): 867-80.

28. Colak H, Dulgergil CT, Dalli M, Hamidi MM. Early childhood caries update: A review of causes, diagnoses, and treatments. J Nat Sci Biol Med. 2013 Jan; 4(1): 29-38.

29. Correa-Faria $P$, Martins-Junior PA, Vieira-Andrade RG, Marques LS, Ramos-Jorge ML. Factors associated with the development of early childhood caries among Brazilian preschoolers. Braz Oral Res. 2013; 27(4): 356-62.

30. Rai NK, Tiwari T. Parental Factors Influencing the Development of Early Childhood Caries in Developing Nations: A Systematic Review. Front public Heal. 2018; 16 (6): 64.

31. Ministerio de Salud Pública del Ecuador. Alimentación y nutrición de la mujer gestante y de la madre en periodo de lactancia. Guía de Práctica Clínica (GPC) [Internet]. Quito. 2014. [Citado: 15 Diciembre 2017] En: http://instituciones.msp.gob.ec/documentos/Guias/guias 2014/Alimentacion y nutricion de la madre 25-11-14.pdf

32. Ministerio de Salud Pública del Ecuador. Modelo de Atención Integral del Sistema Nacional de Salud (MAIS) [Internet]. Quito. 2012. [Citado: 15 Diciembre 2017]. En: http://instituciones.msp.gob.ec/somossalud/images/docum entos/guia/Manual_MAIS-MSP12.12.12.pdf.

33. Ministerio de Inclusión Económica y Social. Desarrollo infantil para el buen vivir, un análisis para la política pública [Internet]. Quito. 2016. [Citado: 10 Diciembre 2017].En: http://www.inclusion.gob.ec/wpcontent/uploads/downloads/2013/11/Libro-de-PolíticasPúblicas.pdf

34. Asamblea Constituyente del Ecuador. Constitución de la República del Ecuador [Internet]. Quito. 2008. [Citado: 13 Diciembre 2017].En: https://www.oas.org/juridico/pdfs/mesicic4_ecu_const.pdf

35. Asamblea Constituyente del Ecuador. Ley Ōrgánica de Salud y su Reglamento -Decreto 1395 [Internet]. Quito. 2008. [Citado: 15 Diciembre 2017]. En: http://extwprlegs1.fao.org/docs/pdf/ecu154958.pdf 